\title{
PRELIMINARY NOTE ON THE DIFFERENTIATION OF ANGIOBLASTS AND THE METHOD BY WHICH THEY PRODUCE BLOOD-VESSELS, BLOOD-PLASMA AND RED BLOOD-CELLS AS SEEN IN THE LIVING CHICK
}

FLORENCE R. SABIN

Anatomical Laboratory, Johns Hopkins University

The question of the origin of the vascular system can be solved by the method of studying the living blastoderm of the chick in hanging-drop preparations.

By watching chicks of the second day of incubation in these preparations it is possible to see all the processes by which blood-vessels and later blood-cells form. These observations can be made best on the area pellucida. Blood-vessels begin by the differentiation from mesoderm of a new type of cells, angioblasts or vaso-formative cells. They differ from mesoderm in having a much more granular cytoplasm and in being more refractile. They differ also in their behavior and in their potentialities. When a cell of the mesoderm divides, the daughter cells separate at least enough so that they can be recognized as distinct cells but angioblasts give daughter cells that remain together to form dense syncytial masses. These small masses soon join similar masses by means of tiny processes of cytoplasm put out from them exactly like the sprouts by which blood-vessels are known to grow. In this way angioblasts form a plexus of dense masses of cells in sharp contrast to the more delicate plexus of mesoderm which represents the early stages of the development of the coelom. The plexus of angioblasts increases both by the division and the growth of its cells and by the constant addition of new angioblasts which differentiate from the mesoderm. 
Within the plexus of angioblasts, vacuoles appear which represent a liquefaction of the central part of the cytoplasm to form blood-plasma. The vacuoles begin against the nuclei and may occur anywhere in the mass but are especially frequent under the edges. The vacuoles just under the margins leave the cells along the border of the mass to become an endothelial lining of a cavity. The complete liquefaction of the central part of the mass into plasma takes from one to two hours and can be seen with great clearness in the living chick. The endothelial cells on the border of these cavities become less granular than the original angioblasts. Since the fluid is formed from the liquefying of the center there is no sign of distention of the cavities and no flattening of the cells along the border. There is a destruction both of the cytoplasm and of the nuclei of the mass to form the plasma.

This process of the liquefaction of the central part of the mass of angioblasts to form vessels takes place not only within the plexus but in masses of angioblasts which are still isolated and in this way small vesicles are formed which then join the main plexus by processes of cytoplasm. I have seen such a tiny vesicle form from a single angioblast proving that the lumen of a blood-vessel is intracellular.

There are two processes from which the formation of bloodvessels must be sharply distinguished in watching these living specimens. The first is the formation of the coelom. The mesoderm of the chick is originally a continuous sheet of cells as is shown in Lilly's figure $40, A$ and $B$, on page $79^{1}$ and in Duval's figures 184 to 188 on plate $12 .{ }^{2}$ As the chick grows larger this double sheet of cells forms a plexus with wide interspaces in the network where the mesoderm is entirely lacking leaving nothing but ectoderm and endoderm. The coelom now begins as clefts full of fluid within the solid bands, often at the nodes of the network of mesoderm. These spaces separate and, as it were, split the two layers of the mesoderm apart

\footnotetext{
${ }^{1}$ Lilly, F. R. 1908 The developmont of the chick. Henry Holt and Company, New York.

2 Duval, M. 1889 Atlas d'Embryologie. G. Masson, Paris.
} 
and gradually flow together to make the cavity of the coelom. The mesoderm on the borders of these spaces gradually flattens into a mesothelium. The process involves but little destruction of tissue. At the stage of about six or seven somites a living specimen thus shows a double plexus over the area pellucida, a dorsal plexus which is the developing coelom with large interspaces in the net and many tiny cavities representing the exo-coelom; and a ventral, more massive plexus of angioblasts with much more granular and more refractile cytoplasm than the mesoderm. The granules of the angioblasts are strongly basophilic and stain intensely with haematoxylin. and with azur.

The second structure from which developing vessels must be distinguished I shall call endodermal blebs or blisters. They are collections of fluid beneath the endoderm, that is between endoderm and mesoderm, which are very frequent in the normal chick. They can be seen in any collection of mounted blastoderms. In the living chick they vary in appearance according to their size and shape. Their margins simulate endothelium to a marked degree. If they are distended and hence round, their margins will be thin, sharp and highly refractile; if they are flatter their walls will be still refractile but thicker. Often their nuclei seem to project into a cavity exactly like those of endothelial cells. They may be numerous, small and isolated or large and multiple reminding one of multilocular cysts. As can be readily imagined from their structure they may change in shape rapidly, far more rapidly than true vessels change. They can be analyzed with the focusing screw both by following their margins over onto the endoderm and by noting their very superficial position. They occur under the ectoderm as well as under the endoderm but I think less frequently. No one can follow the development of blood-vessels in the living chick without becoming thoroughly familiar with the appearances of these blisters. They have nothing to do with blood-vessels occurring both before. the blood-vessels begin and afterward. They are however important physiological structures representing the method by which fluid is absorbed through the endoderm and ectoderm for the young chick. 
Red blood-corpuscles can be seen to grow from the endothelial lining of blood-vessels. They may develop from little masses of the original angioblasts which become partially separated by the liquefaction of cytoplasm around them. Such a mass of cells becomes a blood-island by having haemoglobin develop within the cells. The color of haemoglobin can be detected in the living cells earlier than I have been able to fix and stain it. Again an endothelial cell of a blood-vessel will divide so that one daughter cell projects into the lumen. This cell becomes filled with basophilic granules and develops haemoglobin. It is then a unicellular blood-island. It divides and the mass is increased also by the addition of other cells which differentiate from the endothelium in the neighborhood and creep along the wall to join the first cell. These cells soon form a yellow syncytial mass projecting into the lumen of the vessel. At this stage the islands have a smooth, sharp contour. As they develop, cells begin to round up on their surface until the whole mass comes to look like a mulberry and then the red cells break free from the mass and float away in the blood-plasma.

These blood-islands I have seen develop in all the vessels of the area vasculosa, in the omphalo-mesenteric vein and arteries and in the dorsal aorta. In the area pellucida they are most abundant in the plexus of vessels just posterior to the area in which the omphalo-mesenteric arteries develop. In this area almost any chick of fourteen somites will show a plexus of angioblasts full of vacuoles and one of seventeen somites will show blood-islands. The circulation does not interfere with the development of the islands. If they clog the lumen the free corpuscles either lodge behind them for a time or pass on through other channels in the plexus.

One of the most interesting points about the masses of angioblasts and of the islands is that all of the cells in them divide at about the same time. Moreover all of the separate islands of a given area seem to divide at once. In these total preparations, all one can see of the process of mitosis is the nucleus in the stage of the metaphase, and the actual division of the cytoplasm. When the syncytial mass is about to divide it becomes 
intensely refractile and its cells become outlined; then one sees one nucleus after another pass into the metaphase and finally all of the cells appear to be half as large as the mature cells. The island then becomes large by the growth of all the cells to their original size. The blood-cells keep on dividing after they are free from the islands and after they have begun to circulate.

These studies have been made with chicks which were grown in the mixture of Locke's solution and chicken bouillon developed by Margaret Reed Lewis for tissue-cultures. ${ }^{3}$ For the young blastoderms it is better to increase the amount of the sodium chloride in the solution to 1.06 per cent. A weaker solution lakes the haemoglobin and is also less favorable for all the other cells.

From these studies which are here reported briefly, certain principles are established. Blood-vessels do not arise from the dilatation of tissue-spaces, but by the differentiation of angioblasts. They cannot be spoken of as arising from spaces because they develop within the bodies of these angioblasts; that is, they arise within cells, not between them. The processes by which they form are not in any way similar to the processes by which either the coelom or great systems of tissue-spaces like the cerebro-spinal spaces develop. The coelom forms by the splitting apart of two layers of mesoderm with little destruction of tissue; the cerebro-spinal spaces form in a mass of typical mesenchyme with considerable destruction of tissue. Angioblasts differentiate throughout the wall of the yolk sac and in the embryo as well. I have seen the dorsal aorta differentiate in situ in the living chick even to some extent that part within the head. Angioblasts produce blood-plasma, endothelium and red blood-cells. Angioblasts and later endothelial cells give rise to red blood-cells by developing haemoglobin.

The term blood-island has been used since the time of the early embryologists, notably Wolff and Pander, for the masses of vaso-formative cells which can be seen in the area opaque of the chick even before the first somite. I propose however to

${ }^{3}$ Lewis, M. R. and W. H. 1911 The growth of embryonic chick tissues in artificial media, agar and bouillon. Johnis Hopkins Hospital Bulletin, 22. 
restrict the term blood-island to those masses of cells which can be shown to produce haemoglobin and to become free red bloopd-cells. These masses are attached to the wall and hence are not strictly speaking islands. The more primitive masses which do begin as isolated masses or islands of cells but must first produce endothelium and plasma, I shall call angioblasts. The red blood-cells develop after some plasma has been formed. All of the blood-cells of the chick of the second day of incubation can be seen to have haemoglobin in the living chick and hence they cannot be considered as forerunners of white bloodcells. 